\title{
Internally club and approachable for larger structures
}

by

\author{
John Krueger (Berkeley, CA)
}

\begin{abstract}
We generalize the notion of a fat subset of a regular cardinal $\kappa$ to a fat subset of $P_{\kappa}(X)$, where $\kappa \subseteq X$. Suppose $\mu<\kappa, \mu^{<\mu}=\mu$, and $\kappa$ is supercompact. Then there is a generic extension in which $\kappa=\mu^{++}$, and for all regular $\lambda \geq \mu^{++}$, there are stationarily many $N$ in $[H(\lambda)]^{\mu^{+}}$which are internally club but not internally approachable.
\end{abstract}

Suppose $\mu$ is an infinite cardinal. A set $N$ is internally approachable with length $\mu^{+}$if $N$ is the union of an increasing and continuous sequence $\left\langle N_{i}: i<\mu^{+}\right\rangle$of sets with size $\mu$ such that for all $\alpha<\mu^{+},\left\langle N_{i}: i<\alpha\right\rangle$ is in $N$. A related idea is that of an internally club set. A set $N$ with size $\mu^{+}$ is internally club if $N \cap[N]^{\mu}$ contains a club subset of $[N]^{\mu}$. In other words, $N$ is the union of an increasing and continuous sequence $\left\langle N_{i}: i<\mu^{+}\right\rangle$of sets with size $\mu$ such that each $N_{i}$ is in $N$.

Foreman and Todorčević [3] asked whether the properties of being internally approachable and internally club are equivalent. In [5] we proved that under PFA, for all regular $\lambda \geq \omega_{2}$ there are stationarily many structures $N \prec H(\lambda)$ with size $\aleph_{1}$ such that $N$ is internally club but not internally approachable. In this paper we generalize this result to larger structures.

THEOREM 1. Suppose $\mu<\kappa, \mu^{<\mu}=\mu$, and $\kappa$ is supercompact. Then there is a $\mu$-closed, $\mu^{+}$-proper forcing poset which collapses $\kappa$ to become $\mu^{++}$, and forces that for all regular $\lambda \geq \mu^{++}$, there are stationarily many $N$ in $[H(\lambda)]^{\mu^{+}}$which are internally club but not internally approachable.

In the model we construct to prove Theorem 1 , we have $2^{\mu}=\mu^{++}$. In fact, if $2^{\mu}=\mu^{+}$, then any elementary substructure $N \prec H(\lambda)$ with size $\mu^{+}$ and which contains $\mu^{+}$is internally club iff it is internally approachable; this is shown at the end of the paper.

In Section 1 we review notation and some background material. Section 2 generalizes the idea of a fat subset of a regular cardinal $\kappa$ to a fat subset of

2000 Mathematics Subject Classification: 03E05, 03E35.

Key words and phrases: fat, internally club, internally approachable. 
$P_{\kappa}(X)$, where $\kappa \subseteq X$. Section 3 presents the basic forcing poset we use in our consistency result, and in Section 4 we describe how to iterate this poset with a mixed support forcing iteration. In Section 5 we prove Theorem 1.

1. Preliminaries. If $\kappa$ is regular and $\kappa \subseteq X$, we say $C \subseteq P_{\kappa}(X)$ is $c l u b$ if it is closed under unions of increasing sequences of length less than $\kappa$, and is cofinal. A set $S \subseteq P_{\kappa}(X)$ is stationary if it has non-empty intersection with every club. We will use the fact that if $C \subseteq P_{\kappa}(X)$ is club, and $A \subseteq C$ is a directed set with size less than $\kappa$, then $\bigcup A \in C$ (see Lemma 8.25 of [4] for a proof). By directed we mean that if $a$ and $b$ are in $A$, then there is $c$ in $A$ such that $a \cup b \subseteq c$.

If $N$ is a set, $\mathbb{P}$ is a forcing poset, and $G$ is a filter on $\mathbb{P}$, then $N[G]$ denotes the set $\left\{\dot{a}^{G}: \dot{a} \in N \cap V^{\mathbb{P}}\right\}$. A filter $G$ on $\mathbb{P}$ is $N$-generic if for every dense set $D \subseteq \mathbb{P}$ in $N, N \cap D \cap G$ is non-empty. A condition $q$ in $\mathbb{P}$ is $N$-generic if $q$ forces $\dot{G}$ is $N$-generic, where $\dot{G}$ is a name for the generic filter. Suppose $\lambda$ is regular with $\mathbb{P} \in H(\lambda)$, and $N \prec\langle H(\lambda), \in, \mathbb{P}\rangle$. Then for any condition $q$ in $\mathbb{P}$, the following are equivalent: (1) $q$ is $N$-generic, (2) for every dense set $D \subseteq \mathbb{P}$ in $N, N \cap D$ is predense below $q$, (3) $q$ forces $N[\dot{G}] \cap$ On $=N \cap$ On, and (4) $q$ forces $N[\dot{G}] \cap V=N$. Note that if $q$ is $N$-generic, then for any set $X, q$ forces $N[\dot{G}] \cap \check{X}=N \cap \check{X}$.

Suppose $\mathbb{P}$ is a forcing poset and $\lambda$ is regular with $\mathbb{P} \in H(\lambda)$. If $G$ is generic for $\mathbb{P}$ over $V$, then $H(\lambda)^{V[G]}=H(\lambda)^{V}[G]$. Suppose $N \prec\langle H(\theta), \in, \mathbb{P}\rangle$ in $V$. If $G$ is generic for $\mathbb{P}$ over $V$, then $N[G] \prec H(\theta)^{V[G]}$.

Let $\mathbb{P}$ be a forcing poset and $\mu$ a regular cardinal with $\mu^{<\mu}=\mu$. Then $\mathbb{P}$ is $\mu^{+}$-proper if for any regular cardinal $\theta>2^{|\mathbb{P}|}$ with $\mathbb{P}$ in $H(\theta)$, if $N$ is an elementary substructure of $\langle H(\theta), \in, \mathbb{P}\rangle, N$ has size $\mu$, and $N^{<\mu} \subseteq N$, then for all $p$ in $N \cap \mathbb{P}$, there is $q \leq p$ which is $N$-generic. Any $\mu^{+}$-proper forcing poset preserves $\mu^{+}$. Note that if $\mathbb{P}$ is $\mu^{+}$-c.c. then any condition in $\mathbb{P}$ is $N$-generic, since every maximal antichain of $\mathbb{P}$ in $N$ is actually a subset of $N$.

If $\mu$ is a regular cardinal and $\mathbb{P}$ is a forcing poset, we say $\mathbb{P}$ is $\mu$-distributive if for any collection $\mathcal{D}$ of not more than $\mu$ dense open subsets of $\mathbb{P}, \cap \mathcal{D}$ is dense open. This property is equivalent to $\mathbb{P}$ not adding any new sequences of ordinals with order type less than or equal to $\mu$. If $\kappa$ is a cardinal we say $\mathbb{P}$ is $<\kappa$-distributive if $\mathbb{P}$ is $\mu$-distributive for all regular $\mu<\kappa$.

Let $\mathbb{P}$ be a forcing poset and $\mu$ a regular cardinal. We say $\mathbb{P}$ is $\mu$-closed if whenever $\left\langle p_{i}: i<\xi\right\rangle$ is a descending sequence of conditions in $\mathbb{P}$ with $\xi<\mu$, there is $q$ in $\mathbb{P}$ such that $q \leq p_{i}$ for all $i<\xi$. If $A \subseteq \mathbb{P}$, a greatest lower bound of $A$, or $g l b$ of $A$, is a condition $q$ such that $q \leq p$ for all $p$ in $A$, and whenever $r \leq p$ for all $p$ in $A$, then $r \leq q$. We say $\mathbb{P}$ is $\mu$-glb closed if whenever $\left\langle p_{i}: i<\xi\right\rangle$ is a descending sequence of conditions in $\mathbb{P}$ with $\xi<\mu$, there exists a greatest lower bound for the set $\left\{p_{i}: i<\xi\right\}$. 
2. Generalized fat sets. Let $\kappa$ be a regular uncountable cardinal. Recall that a set $A \subseteq \kappa$ is fat if for any club set $C \subseteq \kappa$ and $\xi<\kappa, A \cap C$ contains a closed subset with order type at least $\xi$.

FACT 2.1 (Abraham and Shelah [1]). Suppose $\kappa$ is strongly inaccessible or $\kappa=\mu^{+}$where $\mu^{<\mu}=\mu$. Then the following are equivalent for a set $A \subseteq \kappa$ :

(1) A is fat.

(2) There is a $<\kappa$-distributive forcing poset $\mathbb{P}$ which forces that $A$ contains a club set.

Suppose $\kappa$ is a regular uncountable cardinal and $\kappa \subseteq X$. We generalize the idea of fatness to subsets of $P_{\kappa}(X)$ with the following definition.

Definition 2.2. Suppose $\kappa$ is a regular uncountable cardinal and $\kappa \subseteq X$. A set $A \subseteq P_{\kappa}(X)$ is fat if for all regular $\theta \geq \kappa$ with $X \subseteq H(\theta)$, for any club $C \subseteq P_{\kappa}(H(\theta))$ and $\xi<\kappa$, there is an increasing and continuous sequence $\left\langle N_{i}: i<\xi\right\rangle$ such that for all $i<\xi, N_{i} \in C, N_{i} \cap X \in A$, and $N_{i} \in N_{i+1}$ when $i+1<\xi$.

Lemma 2.3. Suppose $\kappa=\mu^{+}$. Then $A \subseteq P_{\kappa}(X)$ is fat iff for all regular $\theta \geq \kappa$ with $X \subseteq H(\theta)$, for any club $C \subseteq P_{\kappa}(H(\theta))$, and for any regular cardinal $\lambda \leq \mu$, there is an increasing and continuous sequence $\left\langle N_{i}: i \leq \lambda\right\rangle$ such that for $i \leq \lambda, N_{i} \in C, N_{i} \cap X \in A$, and $N_{i} \in N_{i+1}$ when $i<\lambda$.

Proof. Suppose $A$ satisfies the second condition. Then clearly $A$ is stationary in $P_{\kappa}(X)$. Fix $\theta \geq \kappa$ regular with $X \subseteq H(\theta)$. We prove by induction on $\xi<\mu^{+}$that for any club set $C \subseteq P_{\kappa}(H(\theta))$, there is an increasing and continuous sequence $\left\langle N_{i}: i<\xi\right\rangle$ such that for all $i<\xi, N_{i} \in C, N_{i} \cap X \in A$, and $N_{i} \in N_{i+1}$ when $i+1<\xi$. The successor step of the induction follows from the fact that $A$ is stationary.

Suppose $\delta<\mu^{+}$is a limit ordinal and the claim holds for all $\delta^{\prime}<\delta$. Let $\left\langle\delta_{i}: i<\operatorname{cf}(\delta)\right\rangle$ be increasing and cofinal in $\delta$. Note that $\operatorname{cf}(\delta) \leq \mu$. Let

$$
\mathcal{A}=\left\langle H(\theta), \in,<, X, A, \delta,\left\langle\delta_{i}: i<\operatorname{cf}(\delta)\right\rangle\right\rangle,
$$

where $<$ is a well-ordering of $H(\theta)$. Fix an increasing and continuous sequence $\left\langle N_{i}: i \leq \operatorname{cf}(\delta)\right\rangle$ of sets such that for $i \leq \operatorname{cf}(\delta), N_{i} \in C, N_{i} \prec \mathcal{A}$, $\mu \subseteq N_{i}, N_{i} \cap X \in A$, and $N_{i} \in N_{i+1}$ when $i<\operatorname{cf}(\delta)$.

Fix $i<\operatorname{cf}(\delta)$. By the induction hypothesis, let $\left\langle M_{j}^{i}: j \leq \delta_{i}\right\rangle$ be the $<$-least increasing and continuous sequence with length $\delta_{i}+1$ such that $\mu \cup\left\{N_{i}\right\} \subseteq M_{0}^{i}$, and for $j \leq \delta_{i}, M_{j}^{i} \in C, N_{i} \prec M_{j}^{i}, M_{j}^{i} \cap X \in A$, and $M_{j}^{i} \in M_{j+1}^{i}$ when $j<\delta_{i}$. By elementarity, this sequence is in $N_{i+1}$. Then the set

$$
\left\{N_{i}: i \leq \operatorname{cf}(\delta)\right\} \cup\left\{M_{j}^{i}: i<\operatorname{cf}(\delta), j \leq \delta_{i}\right\},
$$

well-ordered by $\in$, is increasing and continuous with order type at least $\delta$, and for all $N$ in this set, $N \in C$ and $N \cap X \in A$. 
We will now show that our definition of fatness generalizes the classical notion. Indeed, let $A$ be a fat subset of a regular cardinal $\kappa$. We show $A$ is a fat subset of $P_{\kappa}(X)$, where $X=\kappa$, according to Definition 2.2. So let $\theta \geq \kappa$ be regular, and let $C \subseteq P_{\kappa}(H(\theta))$ be club. Fix $\xi<\kappa$. Define by induction an increasing and continuous sequence $\left\langle M_{i}: i<\kappa\right\rangle$ such that for $i<\kappa$, $M_{i} \cap \kappa \in \kappa, M_{i} \in C$, and $M_{i} \in M_{i+1}$. Then $\left\langle M_{i} \cap \kappa: i<\kappa\right\rangle$ is a club subset of $\kappa$. Since $A$ is fat, there is a closed set $a \subseteq \kappa$ with order type at least $\xi$ such that $\left\{M_{i} \cap \kappa: i \in a\right\} \subseteq A$. Then $\left\langle M_{i}: i \in a\right\rangle$ is as required.

Suppose on the other hand that $A \subseteq \kappa$ is fat as a subset of $P_{\kappa}(\kappa)$ by Definition 2.2; we show $A$ is fat as a subset of $\kappa$. Let $C \subseteq \kappa$ be club and fix $\xi<\kappa$. Let $\left\langle N_{i}: i \leq \xi\right\rangle$ be an increasing and continuous sequence of sets in $P_{\kappa}(H(\kappa))$ such that for $i \leq \xi, N_{i} \prec\langle H(\kappa), \in, C\rangle, N_{i} \cap \kappa \in \kappa, N_{i} \cap \kappa \in A$, and $N_{i} \in N_{i+1}$ when $i<\xi$. Then $\left\{N_{i} \cap \kappa: i \leq \xi\right\}$ is a closed set contained in $A \cap C$.

The next theorem generalizes Fact 2.1.

THEOREM 2.4. Suppose $\kappa$ is strongly inaccessible or $\kappa=\mu^{+}$where $\mu^{<\mu}=\mu$. Let $X$ be a set containing $\kappa$. Then the following are equivalent for a set $A \subseteq P_{\kappa}(X)$ :

(1) A is fat.

(2) There is a $<\kappa$-distributive forcing poset which forces there is an increasing and continuous sequence $\left\langle a_{i}: i<\kappa\right\rangle$ which is cofinal in $P_{\kappa}(X)$ such that $a_{i} \in A$ for $i<\kappa$.

Proof. Suppose $A \subseteq P_{\kappa}(X)$ and $\mathbb{P}$ is a $<\kappa$-distributive forcing poset which forces that $\left\langle\dot{a}_{i}: i<\kappa\right\rangle$ is increasing, continuous, and cofinal in $P_{\kappa}(X)$ such that $\dot{a}_{i} \in A$ for $i<\kappa$. We prove that $A$ is fat. So let $\theta \geq \kappa$ be regular with $X \subseteq H(\theta)$. Suppose $C \subseteq P_{\kappa}(H(\theta))$ is club. Let $G$ be generic for $\mathbb{P}$ over $V$, and let $a_{i}=\dot{a}_{i}^{G}$ for $i<\kappa$. Since $\mathbb{P}$ is $<\kappa$-distributive, in $V[G]$ the set $C$ is still a club subset of $P_{\kappa}\left(H(\theta)^{V}\right)$.

We work in $V[G]$. Since $X=\bigcup\left\{a_{i}: i<\kappa\right\}$ and $\left|a_{i}\right|<\kappa$ for all $i<\kappa$, $X$ has size $\kappa$ in the extension. So let $\left\langle x_{i}: i<\kappa\right\rangle$ enumerate $X$. We define by induction an increasing and continuous sequence $\left\langle N_{i}: i<\kappa\right\rangle$ such that for all $i<\kappa, N_{i} \in N_{i+1}$ and $N_{i} \in C$. Choose $N_{0}$ in $C$ arbitrarily. At limits take unions. Suppose $N_{i}$ is defined. Then $N_{i}$ is in $H(\theta)^{V}$, so choose $N_{i+1}$ in $C$ such that $N_{i} \cup\left\{N_{i}\right\} \cup\left\{x_{i}\right\} \subseteq N_{i+1}$. This completes the definition. Now $\left\langle a_{i}: i<\kappa\right\rangle$ and $\left\langle N_{i} \cap X: i<\kappa\right\rangle$ are both club in $P_{\kappa}(X)$. So there is a club $D \subseteq \kappa$ such that for all $i \in D, a_{i}=N_{i} \cap X$. Then $\left\langle N_{i}: i \in D\right\rangle$ is an increasing and continuous sequence such that for all $i \in D, N_{i} \in C$, $N_{i} \in N_{i+1}$, and $N_{i} \cap X \in A$. But every initial segment of this sequence is in $V$ since $\mathbb{P}$ is $<\kappa$-distributive. So $A$ is fat.

In the other direction, suppose $A \subseteq P_{\kappa}(X)$ is fat. Define a forcing poset $\mathbb{P}(A)$ as follows. A condition in $\mathbb{P}(A)$ is an increasing and continuous se- 
quence $\left\langle a_{i}: i \leq \gamma\right\rangle$, where $\gamma<\kappa$, such that $a_{i} \in A$ for all $i \leq \gamma$. The ordering is by extension of sequences. We claim that $\mathbb{P}(A)$ is $<\kappa$-distributive and $\mathbb{P}(A)$ forces that the union of the generic filter is an increasing and continuous sequence cofinal in $P_{\kappa}(X)$ with order type $\kappa$ whose elements are in $A$.

Suppose $\left\langle D_{i}: i<\xi\right\rangle$ is a sequence of dense open subsets of $\mathbb{P}(A)$, where $\xi<\kappa$ is a cardinal. Let $p$ be in $\mathbb{P}(A)$; then we find $q \leq p$ which is in $\bigcap\left\{D_{i}: i<\xi\right\}$. Fix a regular cardinal $\theta \gg \kappa$ with $X \in H(\theta)$, and let

$$
\mathcal{A}=\left\langle H(\theta), \in, X, A, \mathbb{P}(A), p,\left\langle D_{i}: i<\xi\right\rangle\right\rangle .
$$

Since $A$ is fat we can find an increasing and continuous sequence $\left\langle N_{i}: i \leq \xi\right\rangle$ such that for all $i \leq \xi, N_{i} \prec \mathcal{A}, N_{i} \cap \kappa \in \kappa, \xi \subseteq N_{i}, N_{i} \cap X \in A$, and when $i<\xi, N_{i} \in N_{i+1}$.

We define by induction a descending sequence of conditions $\left\langle p_{i}: i \leq \xi\right\rangle$ in $\mathbb{P}(A)$. Our induction hypothesis is that $p_{i}$ is in $N_{i+1}$ and the maximum element of $p_{i}$ is $N_{i} \cap X$. Let $p_{0}=\widehat{p}\left(N_{0} \cap X\right)$. Then $p_{0}$ is a condition, because $p \in N_{0}$ and thus all the elements of $p$ are subsets of $N_{0} \cap X$. Suppose $i<\xi$, and for all $j \leq i, p_{j}$ is defined, $p_{j}$ is a member of $N_{j+1}$, and the maximum element of $p_{j}$ is $N_{j} \cap X$. Since $\xi \subseteq N_{i+1}, D_{i}$ is in $N_{i+1}$. Fix $p_{i}^{*} \leq p_{i}$ in $D_{i} \cap N_{i+1}$. Since $p_{i}^{*}$ has size less than $\kappa$ and $N_{i+1} \cap \kappa \in \kappa$, we have $p_{i}^{*} \subseteq N_{i+1}$, and so every element of $p_{i}^{*}$ is a subset of $N_{i+1}$ as well. Therefore if we let $p_{i+1}=p_{i}^{* \frown}\left(N_{i+1} \cap X\right)$, then $p_{i+1}$ is a condition in $N_{i+2} \cap D_{i}$ below $p_{i}$.

Suppose $\delta \leq \xi$ is a limit ordinal and $p_{i} \in N_{i+1}$ is defined for all $i<\delta$. Let

$$
p_{\delta}=\bigcup\left\{p_{i}: i<\delta\right\} \wedge\left(N_{\delta} \cap X\right),
$$

which is a condition since $N_{\delta} \cap X \in A$ and $N_{\delta}=\bigcup\left\{N_{i}: i<\delta\right\}$. We need to show that $p_{\delta}$ is in $N_{\delta+1}$ when $\delta<\xi$. The sequence $\left\langle p_{i}: i<\delta\right\rangle$ is in $N_{\delta}^{<\xi}$. Since $\kappa$ is either strongly inaccessible or equal to $\mu^{+}$where $\mu^{<\mu}=\mu, N_{\delta}^{<\xi}$ has size less than $\kappa$. But $N_{\delta}^{<\xi} \in N_{\delta+1}$. Since $N_{\delta+1} \cap \kappa \in \kappa, N_{\delta}^{<\xi} \subseteq N_{\delta+1}$. So the sequence $\left\langle p_{i}: i<\delta\right\rangle$ is in $N_{\delta+1}$. Clearly then $p_{\delta}$ is in $N_{\delta+1}$ as well.

This completes the construction of $\left\langle p_{i}: i \leq \xi\right\rangle$. The condition $p_{\xi}$ is below $p$ and is in $\bigcap\left\{D_{i}: i<\xi\right\}$. So $\mathbb{P}(A)$ is $<\kappa$-distributive.

For each $\alpha<\kappa$ let $D_{\alpha}$ be the set of conditions in $\mathbb{P}(A)$ with length at least $\alpha$. Clearly $D_{0}$ is dense open, and if $D_{i}$ is dense open, $D_{i+1}$ is dense open as well. Assume $\delta<\kappa$ is a limit ordinal and $D_{i}$ is dense open for all $i<\delta$. Since $\mathbb{P}(A)$ is $<\kappa$-distributive, $\bigcap\left\{D_{i}: i<\delta\right\}$ is dense open. But if $p$ is in this intersection, $p$ has length at least $\delta$. So $\mathbb{P}(A)$ forces the union of the generic filter has length $\kappa$. By an easy density argument, $\mathbb{P}(A)$ forces the union of the generic filter is cofinal in $P_{\kappa}(X)$.

Since we will use the forcing poset from the last theorem in our consistency proof, we describe it explicitly in the following definition. 
Definition 2.5. Suppose $\kappa$ is regular, $\kappa \subseteq X$, and $A \subseteq P_{\kappa}(X)$ is fat. Let $\mathbb{P}(A)$ be the forcing poset consisting of increasing and continuous sequences $\left\langle a_{i}: i \leq \gamma\right\rangle$, where $\gamma<\kappa$ and $a_{i} \in A$ for $i \leq \gamma$, ordered by extension of sequences.

The forcing poset $\mathbb{P}(A)$ is $<\kappa$-distributive and adds an increasing, continuous, and cofinal sequence $\left\langle a_{i}: i<\kappa\right\rangle$ through $P_{\kappa}(X)$ such that $a_{i} \in A$ for $i<\kappa$. In particular, $\mathbb{P}(A)$ collapses the size of $X$ to be $\kappa$.

If $\kappa=\omega_{1}$ and $\omega_{1} \subseteq X$, one can show using Lemma 2.3 that any stationary set $A \subseteq P_{\omega_{1}}(X)$ is fat. Thus $\mathbb{P}(A)$ is $\omega$-distributive for any stationary set $A \subseteq P_{\omega_{1}}(X)$.

3. The basic forcing poset. We now describe the forcing poset which we will use in our consistency proof.

Suppose $\mu^{<\mu}=\mu$ and $\mu^{+} \subseteq X$. The basic forcing poset we will use is $\operatorname{ADD}(\mu) * \mathbb{P}(\dot{S})$, where $\operatorname{ADD}(\mu)$ adds a Cohen subset to $\mu, \operatorname{ADD}(\mu)$ forces $\dot{S}=[X]^{\mu} \cap V$, and $\mathbb{P}(\dot{S})$ is the forcing poset from Definition 2.5. Thus we need to know that $\operatorname{ADD}(\mu)$ forces $\dot{S}$ is fat. If $\mu^{<\mu}=\mu$ then $\operatorname{ADD}(\mu)$ is $\mu^{+}$-c.c., so this follows from the next proposition.

Proposition 3.1. Suppose $\kappa$ is regular and $\kappa \subseteq X$. Let $\mathbb{P}$ be a $\kappa$-c.c. forcing poset. Then $\mathbb{P}$ forces $P_{\kappa}(X) \cap V$ is fat.

Proof. Let $G$ be generic for $\mathbb{P}$ over $V$. Working in $V[G]$, fix $\theta \geq \kappa$ regular with $X \subseteq H(\theta)$, and let $C \subseteq P_{\kappa}(H(\theta))$ be club. Fix $\chi \gg \theta$ regular such that $H(\chi)$ contains $C$ and $\mathbb{P}$ as members. Recall that $H(\chi)^{V[G]}=H(\chi)^{V}[G]$. Let $\dot{C}$ be a name for $C$ in $H(\chi)^{V}$.

Now back in $V$, define by induction an increasing and continuous sequence $\left\langle N_{i}: i<\kappa\right\rangle$ of elementary substructures of $\langle H(\chi), \in, X, \dot{C}, \mathbb{P}\rangle$ such that for all $i<\kappa,\left|N_{i}\right|<\kappa, N_{i} \cap \kappa \in \kappa$, and $N_{i} \in N_{i+1}$. Then in $V[G]$, for all $i<\kappa, N_{i}[G] \prec\left\langle H(\chi)^{V[G]}, \in, C\right\rangle$. By elementarity, $N_{i}[G] \cap C$ is a directed subset of $C$ with size less than $\kappa$ whose union is equal to $N_{i}[G] \cap H(\theta)$. So $N_{i}[G] \cap H(\theta)$ is in $C$. Since $N_{i} \in N_{i+1}, N_{i}[G] \in N_{i+1}[G]$, and therefore $N_{i}[G] \cap H(\theta) \in N_{i+1}[G] \cap H(\theta)$. But $\mathbb{P}$ is $\kappa$-c.c., so $N_{i}[G] \cap V=N \cap V$. For if $x \in N_{i}[G] \cap V$, there is a name $\dot{x}$ for $x$ in $N_{i}$. The maximal antichain of conditions deciding $\dot{x}$ is in $N_{i}$, and has size less than $\kappa$, so is a subset of $N_{i}$. But then $x$ is in $N_{i}$. In particular, $N_{i}[G] \cap X=N_{i} \cap X$, which is in $P_{\kappa}(X) \cap V$.

The forcing poset $\operatorname{ADD}(\mu)$ is $\mu$-glb closed. Indeed, if $\left\langle p_{i}: i<\xi\right\rangle$ is decreasing in $\operatorname{ADD}(\mu)$ where $\xi<\mu$, then $\bigcup\left\{p_{i}: i<\xi\right\}$ is the greatest lower bound. Note that any two-step forcing iteration of $\mu$-glb closed forcing posets is $\mu$-glb closed. 
Lemma 3.2. Suppose $\mu^{<\mu}=\mu, \mu^{+} \subseteq X$, and $\dot{S}$ is an $\operatorname{ADD}(\mu)$-name for $[X]^{\mu} \cap V$. Then $\operatorname{ADD}(\mu)$ forces that $\mathbb{P}(\dot{S})$ is $\mu$-glb closed. Hence $\operatorname{ADD}(\mu) * \mathbb{P}(\dot{S})$ is $\mu$-glb closed.

Proof. Let $G$ be generic for $\operatorname{ADD}(\mu)$. In $V[G]$, suppose $\left\langle p_{i}: i<\xi\right\rangle$ is a descending sequence of conditions in $\mathbb{P}(S)$ where $\xi<\mu$ is a limit ordinal. For each $i$ write $p_{i}=\left\langle a_{j}: j \leq \gamma_{i}\right\rangle$. Let $\gamma=\sup \left(\left\{\gamma_{i}: i<\xi\right\}\right)$ and $a=\bigcup\left\{a_{i}\right.$ : $i<\gamma\}$. Let

$$
q=\bigcup\left\{p_{i}: i<\xi\right\} \cup\{\langle\gamma, a\rangle\} .
$$

Then $q$ is a condition in $\mathbb{P}(S)$ iff $a$ is in $V$. But since $\operatorname{ADD}(\mu)$ is $\mu$-closed, the sequence $\left\langle a_{\gamma_{i}}: i<\xi\right\rangle$ is in $V$, and hence its union $a$ is in $V$. Clearly any condition which extends each $p_{i}$ must extend $q$, so $q$ is the greatest lower bound of the sequence.

4. Iterating the basic forcing poset. We now describe a mixed support iteration of the forcing poset introduced in the last section.

Fix a cardinal $\mu$ such that $\mu^{<\mu}=\mu$. We consider a forcing iteration

$$
\left\langle\mathbb{P}_{i}, \dot{\mathbb{Q}}_{j}: i \leq \alpha, j<\alpha\right\rangle,
$$

satisfying the following recursive definition:

(1) If $i<\alpha$ is even, $\mathbb{P}_{i}$ forces $\dot{\mathbb{Q}}_{i}=\operatorname{ADD}(\mu)$, and $\mathbb{P}_{i}$ forces $\dot{X}_{i}$ is a set containing $\mu^{+}$.

(2) If $i=j+1<\alpha$ is odd, $\mathbb{P}_{i}$ forces $\dot{S}_{i}=\left[\dot{X}_{j}\right]^{\mu} \cap V\left[\dot{G}_{j}\right]$, where $\dot{G}_{j}$ is a name for the generic filter for $\mathbb{P}_{j}$, and $\dot{\mathbb{Q}}_{i}=\mathbb{P}\left(\dot{S}_{i}\right)$ is the poset from Definition 2.5.

(3) If $i \leq \alpha$ is a limit ordinal, $\mathbb{P}_{i}$ is the poset consisting of partial functions $p: i \rightarrow V$ such that $p \nmid j \in \mathbb{P}_{j}$ for $j<i, \mid \operatorname{dom}(p) \cap\{j<i$ : $j$ even $\} \mid<\mu$, and $\mid \operatorname{dom}(p) \cap\{j<i: j$ odd $\} \mid \leq \mu$.

We assume the following recursion hypotheses for all $\beta<\alpha$, which guarantee that the definition above makes sense.

(4) $\mathbb{P}_{\beta}$ is $\mu$-glb closed and $\mu^{+}$-proper, and so preserves cardinals and cofinalities less than or equal to $\mu^{+}$.

(5) Let $\mathbb{P}_{\beta}^{*}$ be the set of $p$ in $\mathbb{P}_{\beta}$ such that for all even $j$ in $\operatorname{dom}(p)$, there is $x$ in $\operatorname{ADD}(\mu)$ such that $p(j)=\check{x}$. Then $\mathbb{P}_{\beta}^{*}$ is dense in $\mathbb{P}_{\beta}$.

(6) If $\left\langle p_{i}: i<\xi\right\rangle$ is a descending sequence of conditions in $\mathbb{P}_{\beta}^{*}$ with $\xi<\mu$, then the greatest lower bound of this sequence is in $\mathbb{P}_{\beta}^{*}$.

We prove that properties (4)-(6) above also hold for $\mathbb{P}_{\alpha}$.

CASE 1: $\alpha=\beta+1$ is a successor ordinal. We show that $\mathbb{P}_{\alpha}$ is $\mu$-glb closed. This will follow from the fact that a two-step iteration of $\mu$-glb closed forcing posets is $\mu$-glb closed. If $\beta$ is even, then $\mathbb{P}_{\alpha}=\mathbb{P}_{\beta} * \operatorname{ADD}(\mu)$. Since $\mathbb{P}_{\beta}$ is $\mu$-glb closed by recursion, clearly $\mathbb{P}_{\alpha}$ is $\mu$-glb closed as well. Suppose 
$\beta=\gamma+1$ is odd. Then $\mathbb{P}_{\alpha}=\mathbb{P}_{\gamma} * \operatorname{ADD}(\mu) * \mathbb{P}\left(\dot{S}_{\beta}\right)$. By recursion, $\mathbb{P}_{\gamma}$ is $\mu$-glb closed, and by Lemma 3.2, $\mathbb{P}_{\gamma}$ forces that $\operatorname{ADD}(\mu) * \mathbb{P}\left(\dot{S}_{\beta}\right)$ is $\mu$-glb closed. So $\mathbb{P}_{\alpha}$ is $\mu$-glb closed. We prove in Proposition 4.2 below that $\mathbb{P}_{\alpha}$ is $\mu^{+}$-proper.

Now we prove that $\mathbb{P}_{\alpha}^{*}$ is dense in $\mathbb{P}_{\alpha}$. Consider a condition $p$ in $\mathbb{P}_{\alpha}$. If $\beta$ is not in the domain of $p$ or if $\beta$ is odd, fix $q \leq p\left\lceil\beta\right.$ in $\mathbb{P}_{\beta}^{*}$. Then $q \leq p$ is in $\mathbb{P}_{\alpha}^{*}$ if $\beta$ is not in $\operatorname{dom}(p)$, and $q \widehat{q} p(\beta) \leq p$ is in $\mathbb{P}_{\alpha}^{*}$ otherwise. Assume $\beta$ is in $\operatorname{dom}(p)$ and $\beta$ is even. Since $\mathbb{P}_{\beta}$ is $\mu$-closed, it forces that $p(\beta)$ is an element of $\operatorname{ADD}(\mu)$ in the ground model. So choose $r \leq p\left\lceil\beta\right.$ in $\mathbb{P}_{\beta}^{*}$ and $x$ in $\operatorname{ADD}(\mu)$ such that $r$ forces $p(\beta)=\check{x}$. Then $r^{\widehat{x}}$ is as required.

Suppose $\left\langle p_{i}: i<\xi\right\rangle$ is a descending sequence of conditions in $\mathbb{P}_{\alpha}^{*}$ with $\xi<\mu$. We show that the greatest lower bound of this sequence is in $\mathbb{P}_{\alpha}^{*}$. Now $\left\langle p_{i}\lceil\beta: i<\xi\rangle\right.$ is a descending sequence in $\mathbb{P}_{\beta}^{*}$. By induction the greatest lower bound $q$ of this sequence is in $\mathbb{P}_{\beta}^{*}$. If $\beta$ is not in $\operatorname{dom}\left(p_{i}\right)$ for all $i<\xi$, then $q$ is the greatest lower bound of $\left\langle p_{i}: i<\xi\right\rangle$ in $\mathbb{P}_{\alpha}^{*}$. Otherwise let $\gamma<\xi$ be the least ordinal such that $\beta$ is in $\operatorname{dom}\left(p_{\gamma}\right)$. If $\beta$ is odd, let $\dot{u}$ be a $\mathbb{P}_{\beta}$-name for the greatest lower bound of $\left\{p_{i}(\beta): \gamma \leq i<\xi\right\}$. Then $q \widehat{q u}$ is as required. If $\beta$ is even, then fix for each $\gamma \leq i<\xi$ a condition $x_{i}$ in $\operatorname{ADD}(\mu)$ such that $p_{i}(\beta)=\check{x}_{i}$. Let $x=\bigcup\left\{x_{i}: \gamma \leq i<\xi\right\}$. Then $q \widehat{q} \check{x}$ is as required.

CASE 2: $\alpha$ is a limit ordinal. We show that $\mathbb{P}_{\alpha}$ is $\mu$-glb closed. Suppose $\left\langle p_{i}: i<\xi\right\rangle$ is a descending sequence of conditions in $\mathbb{P}_{\alpha}$, with $\xi<\mu$. For each $i<\alpha, \mathbb{P}_{i}$ forces $\dot{\mathbb{Q}}_{i}$ is $\mu$-glb closed. Define $q$ with support equal to $\bigcup\left\{\operatorname{dom}\left(p_{i}\right): i<\xi\right\}$, so that for each $\beta$ in this support, $q \uparrow \beta$ forces $q(\beta)$ is the greatest lower bound of $\left\langle p_{i}(\beta): \gamma_{\beta} \leq i<\xi\right\rangle$, where $\gamma_{\beta}$ is the least $i<\xi$ with $\beta$ in $\operatorname{dom}\left(p_{i}\right)$. Clearly then $q$ is the greatest lower bound of $\left\{p_{i}: i<\xi\right\}$ in $\mathbb{P}_{\alpha}$. Suppose moreover that $p_{i} \in \mathbb{P}_{\alpha}^{*}$ for all $i<\xi$. Then $q$ can be chosen to be in $\mathbb{P}_{\alpha}^{*}$ as well. Namely, for each even $\beta$ in $\operatorname{dom}(q)$, and for $\gamma_{\beta} \leq i<\xi$, choose $x_{i}^{\beta}$ in $\operatorname{ADD}(\mu)$ such that $p_{i}(\beta)=\check{x}_{i}^{\beta}$. Then let $q(\beta)$ be a name for $\bigcup\left\{x_{i}^{\beta}: \gamma_{\beta} \leq i<\xi\right\}$.

Now we show $\mathbb{P}_{\alpha}^{*}$ is dense in $\mathbb{P}_{\alpha}$. First assume $\operatorname{cf}(\alpha) \geq \mu$, and let $p$ be in $\mathbb{P}_{\alpha}$. Then there is $\xi<\alpha$ such that $\operatorname{dom}(p) \cap\{i<\alpha: i$ even $\} \subseteq \xi$. By induction we can choose $q \leq p\left\lceil\xi\right.$ in $\mathbb{P}_{\xi}^{*}$. Then $q \widehat{q} p \uparrow[\xi, \alpha)$ is in $\mathbb{P}_{\alpha}^{*}$ and is below $p$.

Suppose $\operatorname{cf}(\alpha)<\mu$ and let $p$ be in $\mathbb{P}_{\alpha}$. Fix an increasing and continuous sequence $\left\langle\xi_{i}: i<\operatorname{cf}(\alpha)\right\rangle$ cofinal in $\alpha$ with $\xi_{0}=0$, and let $\xi_{\operatorname{cf}(\alpha)}=\alpha$. We define by induction a descending sequence $\left\langle p_{i}: i \leq \operatorname{cf}(\alpha)\right\rangle$ so that $p_{i} \mid \xi_{i}$ is in $\mathbb{P}_{\xi_{i}}^{*}$. Let $p_{0}=p$. Given $p_{i}$, apply the recursion hypotheses to choose

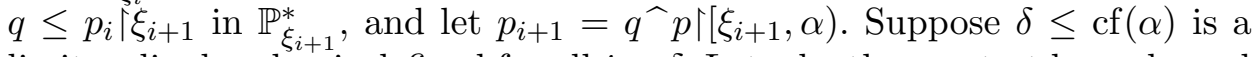
limit ordinal and $p_{i}$ is defined for all $i<\delta$. Let $q$ be the greatest lower bound of the sequence $\left\langle p_{i} \mid \xi_{i}: i<\delta\right\rangle$. Since each $p_{i} \mid \xi_{i}$ is in $\mathbb{P}_{\xi_{i}}^{*} \subseteq \mathbb{P}_{\xi_{\delta}}^{*}, q$ is in $\mathbb{P}_{\xi_{\delta}}^{*}$. Now define $p_{\delta}=q \widehat{\Upsilon} p \uparrow\left[\xi_{\delta}, \alpha\right)$. This completes the definition. The condition $p_{\mathrm{cf}(\alpha)}$ is below $p$ and is in $\mathbb{P}_{\alpha}^{*}$. 
Now we prove that $\mathbb{P}_{\alpha}$ is $\mu^{+}$-proper. The proof is the same whether $\alpha$ is a successor or a limit ordinal.

We will use the following basic observation.

LEMma 4.1. Suppose $p$ and $q$ are conditions in $\mathbb{P}_{\alpha}$ such that for all $\gamma$ in $\operatorname{dom}(p) \cap \operatorname{dom}(q)$, either $p\lceil\gamma$ or $q\lceil\gamma$ forces $p(\gamma)$ and $q(\gamma)$ are compatible in $\dot{\mathbb{Q}}_{\gamma}$. Then $p$ and $q$ are compatible.

Proposition 4.2. The poset $\mathbb{P}_{\alpha}$ is $\mu^{+}$-proper.

Proof. Fix a regular cardinal $\theta>2^{\left|\mathbb{P}_{\alpha}\right|}$ such that $\mathbb{P}_{\alpha}$ is in $H(\theta)$. Let $N \prec\left\langle H(\theta), \in, \mathbb{P}_{\alpha}\right\rangle$ be a set with size $\mu$ with $N^{<\mu} \subseteq N$. We would like to show that for every $p$ in $N \cap \mathbb{P}_{\alpha}$, there is $q \leq p$ which is $N$-generic. In Proposition 4.5 we need $q$ to satisfy a slightly stronger property, which we describe in the following claim.

Claim 4.3. For all $p$ in $N \cap \mathbb{P}_{\alpha}$, there is $q \leq p$ with the property that for all $r \leq q$, and for any dense set $D \subseteq \mathbb{P}_{\alpha}$ in $N$, there is $q^{\prime}$ in $D \cap N$ compatible with $r$ such that for all odd $\gamma$ in $\operatorname{dom}\left(q^{\prime}\right), \gamma \in \operatorname{dom}(r)$ and $r\lceil\gamma$ forces $r(\gamma) \leq q^{\prime}(\gamma)$.

Let $\left\langle\left\langle D_{i}, f_{i}\right\rangle: i<\mu\right\rangle$ be an enumeration of all pairs $\langle D, f\rangle$ in $N$ such that $D \subseteq \mathbb{P}_{\alpha}$ is dense and $f:\{\beta<\alpha: \beta$ even $\} \rightarrow \operatorname{ADD}(\mu)$ is a partial function with $|\operatorname{dom}(f)|<\mu$.

We define by induction a descending sequence $\left\langle p_{i}: i<\mu\right\rangle$ of conditions in $N \cap \mathbb{P}_{\alpha}^{*}$ and a sequence $\left\langle q_{i}: i<\mu\right\rangle$ of conditions in $N \cap \mathbb{P}_{\alpha}^{*}$ such that:

(1) for $i<\mu, \operatorname{dom}\left(p_{i}\right) \cap\{\beta<\alpha: \beta$ even $\}=\operatorname{dom}\left(p_{0}\right) \cap\{\beta<\alpha: \beta$ even $\}$,

(2) for $i<\mu$, for all even $\beta$ in $\operatorname{dom}\left(p_{i}\right), p_{i}(\beta)=p_{0}(\beta)$.

Fix $p_{0} \leq p$ in $N \cap \mathbb{P}_{\alpha}^{*}$. If $\delta<\mu$ is a limit ordinal and $p_{i}$ is defined for all $i<\delta$, let $p_{\delta}$ be the greatest lower bound of $\left\{p_{i}: i<\delta\right\}$. Since $N^{<\mu} \subseteq N$, $\left\langle p_{i}: i<\delta\right\rangle$ is in $N$, and therefore $p_{\delta}$ is in $N \cap \mathbb{P}_{\alpha}^{*}$.

Suppose $p_{i}$ is defined for a fixed $i<\mu$. Consider the pair $\left\langle D_{i}, f_{i}\right\rangle$. If there is $q$ in $N \cap D_{i}$ below $p_{i}$ such that $\operatorname{dom}\left(f_{i}\right) \subseteq \operatorname{dom}(q)$, and for all $\beta$ in $\operatorname{dom}\left(f_{i}\right), q(\beta)$ is a name for $f_{i}(\beta)$, then choose $q_{i}$ as such a $q$. Otherwise just pick $q_{i} \leq p_{i}$ in $N \cap D_{i}$. Now define $p_{i+1}$ with support equal to

$$
\left(\operatorname{dom}\left(p_{i}\right) \cap\{\beta<\alpha: \beta \text { even }\}\right) \cup\left(\operatorname{dom}\left(q_{i}\right) \cap\{\gamma<\alpha: \gamma \operatorname{odd}\}\right)
$$

so that $p_{i+1}(\beta)=p_{i}(\beta)$ for even $\beta$, and $p_{i+1}(\gamma)=q_{i}(\gamma)$ for odd $\gamma$.

We define a lower bound $q$ for $\left\langle p_{i}: i<\mu\right\rangle$, and prove that $q$ satisfies the requirements of Claim 4.3. Clearly then $q$ is $N$-generic. The domain of $q$ is $\bigcup\left\{\operatorname{dom}\left(p_{i}\right): i<\mu\right\}$. In particular, $\operatorname{dom}(q) \cap\{\beta<\alpha: \beta$ even $\}=$ $\operatorname{dom}\left(p_{0}\right) \cap\{\beta<\alpha: \beta$ even $\}$, which has size less than $\mu$. For even $\beta$ in $\operatorname{dom}(q)$, let $q(\beta)=p_{0}(\beta)$.

Suppose $\gamma=\beta+1$ is an odd ordinal in $\operatorname{dom}(q)$. Let $i_{\gamma}<\mu$ be the least $i$ such that $\gamma$ is in $\operatorname{dom}\left(p_{i}\right)$. For $i_{\gamma} \leq i<\mu$, fix a name $\dot{\sigma}_{i}^{\gamma}$ so that $\mathbb{P}_{\gamma}$ forces 
$p_{i}(\gamma)$ has domain $\dot{\sigma}_{i}^{\gamma}+1$. Let $\dot{\sigma}_{\gamma}$ be a $\mathbb{P}_{\gamma}$-name for $\sup \left(\left\{\dot{\sigma}_{i}^{\gamma}+1: i_{\gamma} \leq i<\mu\right\}\right)$. Then $\mathbb{P}_{\gamma}$ forces that the union of the conditions in $\left\{p_{i}(\gamma): i_{\gamma} \leq i<\mu\right\}$ is a sequence of length $\dot{\sigma}_{\gamma}$. Let $\left\langle\dot{a}_{i}^{\gamma}: i<\dot{\sigma}_{\gamma}\right\rangle$ be a sequence of names such that

$$
\mathbb{P}_{\gamma} \Vdash \bigcup\left\{p_{i}(\gamma): i_{\gamma} \leq i<\mu\right\}=\left\langle\dot{a}_{i}^{\gamma}: i<\dot{\sigma}_{\gamma}\right\rangle .
$$

Let $\dot{a}_{\dot{\sigma}_{\gamma}}^{\gamma}$ be a name for $\bigcup\left\{\dot{a}_{i}^{\gamma}: i<\dot{\sigma}_{\gamma}\right\}$. Finally, let $q(\gamma)$ be a name for the sequence $\left\langle\dot{a}_{i}^{\gamma}: i \leq \dot{\sigma}_{\gamma}\right\rangle$.

We prove by induction that for all $\gamma \leq \alpha, q\left\lceil\gamma\right.$ is a condition in $\mathbb{P}_{\gamma}$ and is below $p_{i}\lceil\gamma$ for all $i<\mu$. Limit stages are clear. Suppose $q\lceil\gamma$ satisfies this property. If $\gamma$ is even or if $\gamma$ is not in $\operatorname{dom}(q)$, then clearly $q\lceil\gamma+1$ is as required. Suppose $\gamma=\beta+1$ is odd and is in $\operatorname{dom}(q)$. Then $q\lceil\gamma+1$ is a condition below $p_{i}\left\lceil\gamma+1\right.$ for all $i<\mu$, provided that $q\left\lceil\gamma\right.$ forces that $\dot{a}_{\dot{\sigma}_{\gamma}}^{\gamma}$ is in $\dot{S}_{\gamma}=\left[\dot{X}_{\beta}\right]^{\mu} \cap V\left[\dot{G}_{\beta}\right]$.

Let $G_{\beta} * H$ be generic for $\mathbb{P}_{\gamma}=\mathbb{P}_{\beta} * \operatorname{ADD}(\mu)$. Since $\gamma$ is in $\operatorname{dom}(q)$, $\gamma$ is in $\operatorname{dom}\left(p_{i}\right)$ for some $i<\mu$. Since $\mu \subseteq N$, $\operatorname{dom}\left(p_{i}\right) \subseteq N$. Therefore $\gamma$, and hence $\beta$, is in $N$. So $\mathbb{P}_{\beta}$ is in $N$. But $\operatorname{ADD}(\mu)$ is $\mu^{+}$-c.c. in $V\left[G_{\beta}\right]$, so $N\left[G_{\beta} * H\right] \cap V\left[G_{\beta}\right]=N\left[G_{\beta}\right]$. In particular, $N\left[G_{\beta} * H\right] \cap X_{\beta}=N\left[G_{\beta}\right] \cap X_{\beta}$, which is in $\left[X_{\beta}\right]^{\mu} \cap V\left[G_{\beta}\right]$. So it suffices to show that $a_{\sigma_{\gamma}}^{\gamma}=N\left[G_{\beta} * H\right] \cap X_{\beta}$.

If $i_{\gamma} \leq i<\mu$, then the condition $p_{i}(\gamma)=\left\langle a_{j}^{\gamma}: j \leq \sigma_{i}^{\gamma}\right\rangle$ is a member, and hence a subset, of $N\left[G_{\beta} * H\right]$. Therefore each $a_{j}^{\gamma}$ is a subset of $N\left[G_{\beta} * H\right]$. Hence $\bigcup\left\{a_{j}^{\gamma}: j<\sigma_{\gamma}\right\} \subseteq N\left[G_{\beta} * H\right] \cap X_{\beta}$. On the other hand, fix $x$ in $N\left[G_{\beta} * H\right] \cap X_{\beta}$. Fix a $\mathbb{P}_{\gamma}$-name $\dot{x}$ for $x$ in $N$. Then there is a dense subset of $\mathbb{P}_{\alpha}$ in $N$ of conditions $s$ such that $\mathbb{P}_{\gamma}$ forces $\dot{x}$ is in some element of the sequence $s(\gamma)$. Hence for some $i<\mu, q_{i}$ is in this dense set. Since $q_{i}(\gamma)=p_{i+1}(\gamma), \mathbb{P}_{\gamma}$ forces $\dot{x}$ appears in some element of $p_{i+1}(\gamma)$. Therefore $x$ appears in some element of $\left\langle a_{j}^{\gamma}: j \leq \sigma_{i+1}^{\gamma}\right\rangle$. So $x$ is in $a_{\sigma_{\gamma}}^{\gamma}$. Thus $a_{\sigma_{\gamma}}^{\gamma}=$ $\bigcup\left\{a_{i}^{\gamma}: i<\sigma_{\gamma}\right\}=N\left[G_{\beta} * H\right] \cap X_{\beta}$.

We now prove that $q$ has the property described in Claim 4.3. Let $r \leq q$ and suppose $D$ is a dense subset of $\mathbb{P}_{\alpha}$ in $N$. Fix $s \leq r$ in $\mathbb{P}_{\alpha}^{*} \cap D$. Let $f: \alpha \rightarrow \operatorname{ADD}(\mu)$ be the partial function with $\operatorname{dom}(f)=N \cap \operatorname{dom}(s) \cap$ $\{\beta<\alpha: \beta$ even $\}$ such that for all $\beta$ in $\operatorname{dom}(f), s(\beta)$ is a name for $f(\beta)$. Since $N^{<\mu} \subseteq N, f$ is in $N$. Fix $i<\mu$ such that $D_{i}=D$ and $f_{i}=f$.

Now $H(\theta)$ models that there is $u \leq p_{i}$ in $D_{i}$ such that $\operatorname{dom}\left(f_{i}\right) \subseteq \operatorname{dom}(u)$, and for all $\beta$ in $\operatorname{dom}\left(f_{i}\right), u(\beta)$ is a name for $f_{i}(\beta)$, as witnessed by $u=s$. By elementarity, the same is true in $N$. Hence, by construction, $q_{i}$ also has this property. If $\gamma$ is odd and is in $\operatorname{dom}\left(q_{i}\right)$, then $\gamma$ is in $\operatorname{dom}\left(p_{i+1}\right)$ and $p_{i+1}(\gamma)=q_{i}(\gamma)$. Therefore, for all odd $\gamma$ in $\operatorname{dom}\left(q_{i}\right), \gamma$ is in $\operatorname{dom}(r)$ and $r\lceil\gamma$ forces $r(\gamma) \leq q_{i}(\gamma)$.

We show that $q_{i}$ and $r$ are compatible, which finishes the proof. We apply Lemma 4.1 to show $q_{i}$ and $s$ are compatible. Suppose $\gamma$ is in $\operatorname{dom}\left(q_{i}\right) \cap$ $\operatorname{dom}(s)$. Since $\operatorname{dom}\left(q_{i}\right) \subseteq N, \gamma$ is in $N \cap \operatorname{dom}(s)$. So if $\gamma$ is even, then $\gamma$ is in 
$\operatorname{dom}\left(f_{i}\right)$. Then $q_{i}(\gamma)$ and $s(\gamma)$ are both names for $f_{i}(\gamma)$ and thus are equal. If $\gamma$ is odd, then $q_{i}(\gamma)=p_{i+1}(\gamma)$, and $s\left\lceil\gamma\right.$ forces $s(\gamma) \leq p_{i+1}(\gamma)$.

This completes the recursion.

The next proposition describes a special property of $\mathbb{P}_{\alpha}$ which we will use in the consistency proof of the next section. First we need a technical lemma.

Lemma 4.4. Let $p^{\prime}$ and $q^{\prime}$ be conditions in $\mathbb{P}_{\alpha}^{*}$. Then there are $p \leq p^{\prime}$ and $q \leq q^{\prime}$ in $\mathbb{P}_{\alpha}^{*}$ such that $\operatorname{dom}(p) \cap\{\gamma<\alpha: \gamma$ odd $\}=\operatorname{dom}(q) \cap\{\gamma<\alpha: \gamma$ odd $\}$, and for all odd $\gamma$ in this set, $p(\gamma)=q(\gamma)$.

Proof. First choose $p(0) \leq p^{\prime}(0)$ and $q(0) \leq q^{\prime}(0)$ in $\operatorname{ADD}(\mu)$ which are incompatible. Suppose $\beta>0$ is an even ordinal and $p\lceil\beta$ and $q \uparrow \beta$ are defined. Let $\beta$ be in $\operatorname{dom}(p)$ iff $\beta$ is in $\operatorname{dom}\left(p^{\prime}\right)$, in which case $p(\beta)=p^{\prime}(\beta)$, and similarly with $q$. Suppose $\gamma$ is odd and $p\lceil\gamma$ and $q\lceil\gamma$ are defined. If $\gamma$ is in $\operatorname{dom}\left(p^{\prime}\right) \backslash \operatorname{dom}\left(q^{\prime}\right)$ then let $p(\gamma)=q(\gamma)=p^{\prime}(\gamma)$, and similarly if $\gamma$ is in $\operatorname{dom}\left(q^{\prime}\right) \backslash \operatorname{dom}\left(p^{\prime}\right)$. Suppose $\gamma$ is in $\operatorname{dom}\left(p^{\prime}\right) \cap \operatorname{dom}\left(q^{\prime}\right)$. Let $\dot{x}_{\gamma}$ be a $\mathbb{P}_{\gamma}$-name such that $\mathbb{P}_{\gamma}$ forces $\dot{x}_{\gamma}=p^{\prime}(\gamma)$ if $p\left\lceil\gamma\right.$ is in $\dot{G}_{\gamma}$, and $\dot{x}_{\gamma}=q^{\prime}(\gamma)$ otherwise. Then $\dot{x}_{\gamma}$ is well-defined because $p\lceil\gamma$ and $q\lceil\gamma$ are incompatible. Let $p(\gamma)=q(\gamma)=\dot{x}_{\gamma}$.

Proposition 4.5. The poset $\mathbb{P}_{\alpha}$ forces that whenever $f: \mu^{+} \rightarrow V$ is a function in the extension such that for all $i<\mu^{+}, f\lceil i$ is in $V$, then $f$ is in $V$.

Proof. Suppose for a contradiction that $p$ forces $\dot{f}: \mu^{+} \rightarrow V$ is a function which is not in $V$, but for all $i<\mu^{+}, \dot{f}\lceil i$ is in $V$.

Fix a regular cardinal $\theta \gg \mu^{+}$with $\mathbb{P}_{\alpha} \in H(\theta)$. Let $N$ be an elementary substructure of $\left\langle H(\theta), \in, \mathbb{P}_{\alpha}, p, \dot{f}\right\rangle$ with size $\mu$ and $N^{<\mu} \subseteq N$. By Claim 4.3, fix $q \leq p$ such that for all $r \leq q$ and for any dense set $D \subseteq \mathbb{P}_{\alpha}$ in $N$, there is $q^{\prime}$ in $D \cap N$ compatible with $r$ such that for all odd $\gamma$ in $\operatorname{dom}\left(q^{\prime}\right), \gamma \in \operatorname{dom}(r)$ and $r\left\lceil\gamma\right.$ forces $r(\gamma) \leq q^{\prime}(\gamma)$.

Let $r \leq q$ be in $\mathbb{P}_{\alpha}^{*}$ such that $r$ decides $\dot{f}\left\lceil N \cap \mu^{+}\right.$. Let $g: \alpha \rightarrow \operatorname{ADD}(\mu)$ be the partial function with domain equal to $N \cap \operatorname{dom}(r) \cap\{\beta<\alpha: \beta$ even $\}$ such that for all $\beta$ in $\operatorname{dom}(g), r(\beta)$ is a name for $g(\beta)$. Since $N^{<\mu} \subseteq N, g$ is in $N$.

Define $D$ as the set of $s_{0} \leq p$ in $\mathbb{P}_{\alpha}^{*}$ for which there exists $s_{1}$ in $\mathbb{P}_{\alpha}^{*}$ such that:

(1) $\operatorname{dom}(g) \subseteq \operatorname{dom}\left(s_{0}\right)$,

(2) there is $i<\mu^{+}$and distinct $a_{0}$ and $a_{1}$ such that $s_{0} \Vdash \dot{f}(i)=a_{0}$ and $s_{1} \Vdash \dot{f}(i)=a_{1}$,

(3) $\operatorname{dom}\left(s_{0}\right) \cap\{\gamma<\alpha: \gamma \operatorname{odd}\}=\operatorname{dom}\left(s_{1}\right) \cap\{\gamma<\alpha: \gamma$ odd $\}$,

(4) for all odd $\gamma$ in $\operatorname{dom}\left(s_{0}\right), s_{0}(\gamma)=s_{1}(\gamma)$, 
(5) $\operatorname{dom}(g) \subseteq \operatorname{dom}\left(s_{1}\right)$, and for all $\beta$ in $\operatorname{dom}(g)$, if $g(\beta)$ is compatible with the condition named by $s_{0}(\beta)$, then $s_{1}(\beta)$ is the name for a condition extending $g(\beta)$.

By elementarity, $D$ is in $N$.

We claim that $D$ is dense below $p$. So let $s \leq p$. Extend $s$ to $s^{\prime}$ in $\mathbb{P}_{\alpha}^{*}$ so that $\operatorname{dom}(g) \subseteq \operatorname{dom}\left(s^{\prime}\right)$. Now define $s^{\prime \prime} \leq s^{\prime}$ with the same domain as $s^{\prime}$ as follows. For $\beta \in \operatorname{dom}\left(s^{\prime}\right) \backslash \operatorname{dom}(g)$, let $s^{\prime \prime}(\beta)=s^{\prime}(\beta)$. Suppose $\beta$ is in $\operatorname{dom}(g)$. If $s^{\prime}(\beta)$ names a condition in $\operatorname{ADD}(\mu)$ compatible with $g(\beta)$, let $s^{\prime \prime}(\beta)$ be a name for a condition which extends $g(\beta)$ and $s^{\prime}(\beta)$. Otherwise let $s^{\prime \prime}(\beta)=s^{\prime}(\beta)$.

Since $\dot{f}$ is not in $V$, there is $i<\mu^{+}$such that $s^{\prime \prime}$ does not decide $\dot{f}(i)$. Fix $s_{0}^{\prime}, s_{1}^{\prime} \leq s^{\prime \prime}$ in $\mathbb{P}_{\alpha}^{*}$ and distinct $a_{0}$ and $a_{1}$ so that $s_{0}^{\prime} \Vdash \dot{f}(i)=a_{0}$ and $s_{1}^{\prime} \Vdash \dot{f}(i)=a_{1}$. Now apply Lemma 4.4 to obtain $s_{0} \leq s_{0}^{\prime}$ and $s_{1} \leq s_{1}^{\prime}$ in $\mathbb{P}_{\alpha}^{*}$ satisfying (3) and (4). We check that (5) holds. If $\beta$ is in $\operatorname{dom}(g)$ and $s_{0}(\beta)$ names a condition compatible with $g(\beta)$, then clearly $s^{\prime}(\beta)$ names a condition compatible with $g(\beta)$. So $s^{\prime \prime}(\beta)$ is a name for a condition refining $g(\beta)$. Since $s_{1} \leq s^{\prime \prime}, s_{1}(\beta)$ is a name for a condition refining $g(\beta)$.

By the genericity property of $q$, we can fix $s_{0} \in D \cap N$ which is compatible with $r$, and such that for all odd $\gamma$ in $\operatorname{dom}\left(s_{0}\right), \gamma$ is in $\operatorname{dom}(r)$ and $r\lceil\gamma$ forces that $r(\gamma) \leq s_{0}(\gamma)$. Fix $s_{1}, i, a_{0}$, and $a_{1}$ in $N$ as described in the definition of $D$. Since $r$ decides $\dot{f}(i)$ and $r$ and $s_{0}$ are compatible, $r$ forces $\dot{f}(i)=a_{0}$. So $r$ and $s_{1}$ are incompatible. We will get a contradiction by showing $r$ and $s_{1}$ are compatible.

We apply Lemma 4.1. Suppose $\beta$ is in $\operatorname{dom}(r) \cap \operatorname{dom}\left(s_{1}\right)$ and $\beta$ is even. Then $\beta$ is in $N$, so $\beta$ must be in $\operatorname{dom}(g)$. Since $r$ and $s_{0}$ are compatible, $r(\beta)$ and $s_{0}(\beta)$ are compatible. By $(5), s_{1}(\beta)$ is the name for a condition extending $g(\beta)$. Suppose $\gamma$ is in $\operatorname{dom}(r) \cap \operatorname{dom}\left(s_{1}\right)$ and $\gamma$ is odd. Then $\gamma$ is in $\operatorname{dom}\left(s_{0}\right)$. So $\gamma$ is in $\operatorname{dom}(r)$, and $r\left\lceil\gamma\right.$ forces $r(\gamma) \leq s_{0}(\gamma)$. But $s_{0}(\gamma)=s_{1}(\gamma)$.

5. The consistency result. Suppose $\mu<\kappa$ are cardinals, $\mu^{<\mu}=\mu$, and $\kappa$ is supercompact. We define a forcing iteration of the form given in the last section which collapses $\kappa$ to become $\mu^{++}$, and forces that for all regular $\lambda \geq \mu^{++}$, there are stationarily many $N$ in $[H(\lambda)]^{\mu^{+}}$such that $N$ is internally club but not internally approachable.

Fix a Laver function $f: \kappa \rightarrow V_{\kappa}$. So for all $x$ and $\lambda$, there is an elementary embedding $j: V \rightarrow M$ with critical point $\kappa$ such that $M^{\lambda} \subseteq M$ and $j(f)(\kappa)=x$.

We define by recursion a forcing iteration

$$
\left\langle\mathbb{P}_{i}, \dot{\mathbb{Q}}_{j}: i \leq \kappa, j<\kappa\right\rangle .
$$

Suppose $\mathbb{P}_{i}$ is defined for a fixed $i<\kappa$. If $i$ is an even ordinal, let $\dot{\mathbb{Q}}_{i}$ be a $\mathbb{P}_{i}$-name for $\operatorname{ADD}(\mu)$. Suppose $i=j+1$ is odd. If $f(j)$ is a $\mathbb{P}_{j}$-name for a set 
which contains $\mu^{+}$, let $\dot{X}_{j}=f(j)$, and otherwise let $\dot{X}_{j}$ be a $\mathbb{P}_{j}$-name for $\mu^{+}$. Let $\dot{S}_{i}$ be a $\mathbb{P}_{i}$-name for $\left[\dot{X}_{j}\right]^{\mu} \cap V\left[\dot{G}_{j}\right]$, and let $\dot{\mathbb{Q}}_{i}$ be a $\mathbb{P}_{i}$-name for the poset $\mathbb{P}\left(\dot{S}_{i}\right)$ from Definition 2.5. Suppose $\delta \leq \kappa$ is a limit ordinal and $\mathbb{P}_{i}$ is defined for all $i<\delta$. Then let $\mathbb{P}_{\delta}$ be the poset consisting of all partial functions $p: \delta \rightarrow V$ such that $p\left\lceil i \in \mathbb{P}_{i}\right.$ for all $i<\delta, \mid \operatorname{dom}(p) \cap\{i<\delta: i$ even $\} \mid<\mu$, and $\mid \operatorname{dom}(p) \cap\{i<\delta: i$ odd $\} \mid \leq \mu$.

Since $f$ is a Laver function, there are stationarily many $\alpha<\kappa$ such that $f(\alpha)$ is a $\mathbb{P}_{\alpha}$-name and $\mathbb{P}_{\alpha}$ forces $f(\alpha)=\left(\mu^{++}\right)^{V\left[\dot{G}_{\alpha}\right]}$. Indeed, let $\dot{x}$ be a $\mathbb{P}_{\kappa}$-name for $\left(\mu^{++}\right)^{V\left[\dot{G}_{\kappa}\right]}$. Choose $j: V \rightarrow M$ with critical point $\kappa$ such that $j(f)(\kappa)=\dot{x}$ and $M$ is sufficiently closed that it models $\mathbb{P}_{\kappa}=j\left(\mathbb{P}_{\kappa}\right)\lceil\kappa$ forces $\dot{x}=\left(\mu^{++}\right)^{M\left[\dot{G}_{\kappa}\right]}$. If $C$ is club in $\kappa$, then $\kappa \in j(C)$. Hence by elementarity, there is $\alpha<\kappa$ in $C$ such that $f(\alpha)$ is as desired. But then $\mathbb{P}_{\alpha+2}$ forces $\left|\left(\mu^{++}\right)^{V\left[\dot{G}_{\alpha}\right]}\right|=\mu^{+}$. So $\mathbb{P}_{\kappa}$ collapses all cardinals in the interval $\left(\mu^{+}, \kappa\right)$.

Since $\left|\mathbb{P}_{i}\right|<\kappa$ for all $i<\kappa$, there are club many $\delta<\kappa$ such that $\left|\mathbb{P}_{i}\right|<\delta$ for all $i<\delta$. Suppose $\mu^{+}<\delta \leq \kappa$ is inaccessible and has this property. Then $\mathbb{P}_{\delta}$ is the direct limit of $\left\langle\mathbb{P}_{i}: i<\delta\right\rangle$, where each $\mathbb{P}_{i}$ has size less than $\delta$, and there are stationarily many $\alpha<\delta$ such that $\mathbb{P}_{\alpha}$ is the direct limit of $\left\langle\mathbb{P}_{i}: i<\alpha\right\rangle$. By a standard $\Delta$-system argument, $\mathbb{P}_{\delta}$ is $\delta$-c.c. (see Theorem 2.2 of [2]). In particular, $\mathbb{P}_{\kappa}$ is $\kappa$-c.c. and forces that $\kappa=\mu^{++}$.

Let $G_{\kappa}$ be generic for $\mathbb{P}_{\kappa}$. In $V\left[G_{\kappa}\right]$ let $\lambda \geq \mu^{++}$be regular. In $V$ let $\theta=\left(2^{\lambda}\right)^{+}$. Let $j: V \rightarrow M$ be an elementary embedding with critical point $\kappa$ such that $M^{\theta} \subseteq M$ and $j(f)(\kappa)$ is a $\mathbb{P}_{\kappa}$-name for $H(\lambda)^{V\left[\dot{G}_{\kappa}\right]}$. Then by choice of $j$,

$$
j\left(\mathbb{P}_{\kappa}\right)=\mathbb{P}_{\kappa} * \operatorname{ADD}(\mu) * \mathbb{P}(\dot{S}) * \mathbb{P}_{\text {tail }}
$$

where

$$
\mathbb{P}_{\kappa+1} \Vdash \dot{S}=\dot{S}_{\kappa+1}=\left[H(\lambda)^{V\left[\dot{G}_{\kappa}\right]}\right]^{\mu} \cap M\left[\dot{G}_{\kappa}\right],
$$

and $\mathbb{P}_{\text {tail }}$ is forced to be an iteration of the form given in the previous section. Let $H * K * G_{\text {tail }}$ be generic over $V\left[G_{\kappa}\right]$ for $\operatorname{ADD}(\mu) * \mathbb{P}(\dot{S}) * \mathbb{P}_{\text {tail }}$. Extend $j$ in $V\left[G_{\kappa} * H * K * G_{\text {tail }}\right]$ to

$$
j: V\left[G_{\kappa}\right] \rightarrow M\left[G_{\kappa} * H * K * G_{\text {tail }}\right] .
$$

Then $j\left(G_{\kappa}\right)=G_{\kappa} * H * K * G_{\text {tail }}$. Since $\mathbb{P}_{\kappa}$ is $\kappa$-c.c., $M\left[G_{\kappa}\right]^{\theta} \cap V\left[G_{\kappa}\right] \subseteq M\left[G_{\kappa}\right]$. In particular, $H(\lambda)^{V\left[G_{\kappa}\right]}=H(\lambda)^{M\left[G_{\kappa}\right]}$.

Working in $V\left[G_{\kappa}\right]$, let $C \subseteq[H(\lambda)]^{\mu^{+}}$be club. We prove there is a set in $C$ which is internally club but not internally approachable. By elementarity, it suffices to prove the same statement about $j(C)$ in $M\left[j\left(G_{\kappa}\right)\right]$. We will prove that in $M\left[j\left(G_{\kappa}\right)\right]$, the set $j " H(\lambda)^{V\left[G_{\kappa}\right]}$ is in $j(C)$ and is internally club but not internally approachable.

Let $N^{*}=j^{\prime \prime} H(\lambda)^{V\left[G_{\kappa}\right]}$. First we prove that $N^{*}$ is in $M\left[j\left(G_{\kappa}\right)\right]$. The set $j^{\text {" }} H(\lambda)^{V}$ is in $M$ by the closure of $M$. But $H(\lambda)^{V\left[G_{\kappa}\right]}=H(\lambda)^{V}\left[G_{\kappa}\right]$. So every 
element of $N^{*}$ is of the form $j\left(\dot{a}^{G_{\kappa}}\right)=j(\dot{a})^{j\left(G_{\kappa}\right)}$, where $\dot{a}$ is in $H(\lambda)^{V}$. So $N^{*}=\left(j\right.$ " $\left.H(\lambda)^{V}\right)\left[j\left(G_{\kappa}\right)\right]$, which is in $M\left[j\left(G_{\kappa}\right)\right]$. Also note that in $M\left[j\left(G_{\kappa}\right)\right]$, $\left|N^{*}\right|=\left|H(\lambda)^{V\left[G_{\kappa}\right]}\right|=\mu^{+}$.

We claim that $N^{*}$ is in $j(C)$. Since $j(C)$ is closed under unions of directed subsets with size less than $j\left(\mu^{++}\right)$, it suffices to show that $N^{*} \cap j(C)$ is directed and $\bigcup\left(N^{*} \cap j(C)\right)=N^{*}$. Suppose $j(a)$ and $j(b)$ are in $N^{*} \cap j(C)$. By elementarity, $a$ and $b$ are in $C$. Fix $c$ in $C$ such that $a \cup b \subseteq c$. Then $j(a)$ and $j(b)$ are contained in $j(c)$ and $j(c) \in N^{*} \cap j(C)$. Hence $N^{*} \cap j(C)$ is directed.

We show that $\bigcup\left(N^{*} \cap j(C)\right)=N^{*}$. Let $j(x)$ be in $N^{*}$. Then $x$ is in $H(\lambda)^{V\left[G_{\kappa}\right]}$, so there is $a$ in $C$ such that $x \in a$. Then $j(x) \in j(a) \in N^{*} \cap j(C)$. So $N^{*} \subseteq \bigcup\left(N^{*} \cap j(C)\right)$. On the other hand, suppose $y$ is in $\bigcup\left(N^{*} \cap j(C)\right)$. Fix $j(a) \in N^{*} \cap j(C)$ so that $y \in j(a)$. Then $a$ is in $C$. In $V\left[G_{\kappa}\right], a$ has size less than $\mu^{++}=\kappa$, and $\kappa$ is the critical point of $j$. So $j(a)=j$ " $a$, and clearly $j$ " $a \subseteq N^{*}$. Thus $y$ is in $N^{*}$. Therefore $N^{*}=\bigcup\left(N^{*} \cap j(C)\right)$ and $N^{*}$ is in $j(C)$.

Now we show that $N^{*}$ is internally club but not internally approachable. Let $N=H(\lambda)^{V\left[G_{\kappa}\right]}$. Since $N$ is transitive and isomorphic to $N^{*}$ by the map $j\left\lceil N, N\right.$ is the transitive collapse of $N^{*}$ and $j^{-1}\left\lceil N^{*}=\pi\right.$ is the transitive collapse map.

Recall that $H * K$ is generic for $\operatorname{ADD}(\mu) * \mathbb{P}(\dot{S})$ over $M\left[G_{\kappa}\right]$, and $S=$ $\dot{S}^{H}=[N]^{\mu} \cap M\left[G_{\kappa}\right]$. Write $\bigcup K=\left\langle a_{i}: i<\mu^{+}\right\rangle$. Then $N=\bigcup\left\{a_{i}: i<\mu^{+}\right\}$. For all $i<\mu^{+}, a_{i}$ is a subset of $N=H(\lambda)^{M\left[G_{\kappa}\right]}$ which is in $M\left[G_{\kappa}\right]$, and $a_{i}$ has size $\mu$, which is less than $\lambda$. Therefore $a_{i}$ is in $H(\lambda)^{M\left[G_{\kappa}\right]}=N$. Hence $N$ is internally club. But then $\left\langle j\left(a_{i}\right): i\left\langle\mu^{+}\right\rangle=\left\langle j^{\text {" }} a_{i}: i\left\langle\mu^{+}\right\rangle\right.\right.$witnesses that $N^{*}$ is internally club.

Suppose for a contradiction that $N^{*}$ is internally approachable in $M\left[j\left(G_{\kappa}\right)\right]$, as witnessed by a sequence $\left\langle N_{i}^{*}: i<\mu^{+}\right\rangle$. Note that $N$ is then also internally approachable. Indeed, for all $i<\mu^{+}$, let $N_{i}=\pi\left(N_{i}^{*}\right)=\pi^{\text {" }}\left(N_{i}^{*}\right)$. Clearly, $\left\langle N_{i}: i\left\langle\mu^{+}\right\rangle\right.$is increasing and continuous and its union is equal to $N$. For each $\alpha<\mu^{+}$, choose $f_{\alpha}$ in $N$ such that $j\left(f_{\alpha}\right)=\left\langle N_{i}^{*}: i<\alpha\right\rangle$. Then for $i<\alpha, j\left(N_{i}\right)=N_{i}^{*}=j\left(f_{\alpha}\right)(i)=j\left(f_{\alpha}\right)(j(i))=j\left(f_{\alpha}(i)\right)$. So $N_{i}=f_{\alpha}(i)$. Therefore $\left\langle N_{i}: i\langle\alpha\rangle=f_{\alpha}\right.$, which is in $N$. Hence $\left\langle N_{i}: i<\mu^{+}\right\rangle$witnesses that $N$ is internally approachable.

Let $f=\left\langle N_{i}: i<\mu^{+}\right\rangle$. Then for all $i<\mu^{+}, f\left\lceil i\right.$ is in $N$. Since $N \subseteq M\left[G_{\kappa}\right]$, for all $i<\mu^{+}, f\left\lceil i\right.$ is in $M\left[G_{\kappa}\right]$. By Proposition 4.5, $f=\left\langle N_{i}: i<\mu^{+}\right\rangle$is in $M\left[G_{\kappa}\right]$. But this implies $N$ has size $\mu^{+}$in $M\left[G_{\kappa}\right]$, which is false. This completes the proof of Theorem 1.

We note that if GCH holds in $V$, then in $V[G], 2^{\mu}=\mu^{++}$and $2^{\alpha}=\alpha^{+}$for all infinite cardinals $\alpha$ different from $\mu$. This violation of $\mathrm{GCH}$ is necessary, by the following argument: 
Suppose $2^{\mu}=\mu^{+}$and $\lambda \geq \mu^{++}$is regular. Let $N \prec H(\lambda)$ be a model with size $\mu^{+}$such that $\mu^{+} \subseteq N$, and $N$ has the $\mu^{+}$-covering property, that is, every subset of $N$ with size less than $\mu^{+}$is a subset of a member of $N$ with size less than $\mu^{+}$. Then $N^{\mu} \subseteq N$. For if $a \subseteq N$ has size $\mu$, then $a$ is covered by a set $b$ in $N$ with size $\mu$. Since $2^{\mu}=\mu^{+}$, we can enumerate the power set of $b$ by a sequence $\left\langle x_{i}: i<\mu^{+}\right\rangle$in $N$. But $\mu^{+} \subseteq N$, so $x_{i} \in N$ for all $i<\mu^{+}$. In particular, $a$ is in $N$. Now fix an increasing and continuous sequence $\left\langle N_{i}: i<\mu^{+}\right\rangle$of sets with size $\mu$ whose union is $N$. Since $N^{\mu} \subseteq N$, each $N_{i}$ is in $N$, and thus every initial segment of this sequence is in $N$. So $N$ is internally approachable. But if $N$ is internally club, then $N$ has the $\mu^{+}$-covering property.

Remarks. Mixed support iterations similar to that presented in Section 4 appear in Chapter 8 of [8], where an analogue of Proposition 4.2 is proved for iterations of posets of the form $\mathbb{P} * \dot{\mathbb{Q}}$, where $\mathbb{P}$ is $\omega_{1}$-closed, $\mathbb{P}$ satisfies a strengthening of $\omega_{2}$-c.c., and $\dot{\mathbb{Q}}$ is forced to be $\omega_{2}$-closed. The proof of our consistency result is related to Mitchell's construction in [6] of a model with no Aronszajn trees on $\omega_{2}$. See [7] for a recent discussion concerning the special property described in Proposition 4.5.

\section{References}

[1] U. Abraham and S. Shelah, Forcing closed unbounded sets, J. Symbolic Logic 48 (1983), 643-657.

[2] J. Baumgartner, Iterated forcing, in: Surveys in Set Theory, Cambridge Univ. Press, 1983, 1-59.

[3] M. Foreman and S. Todorčević, A new Löwenheim-Skolem theorem, Trans. Amer. Math. Soc. 357 (2005), 1693-1715.

[4] T. Jech, Set Theory, 3rd ed., Springer, 2003.

[5] J. Krueger, Internally club and approachable, Adv. Math. 213 (2007), 734-740.

[6] W. Mitchell, Aronszajn trees and the independence of the transfer property, Ann. Math. Logic 5 (1972), 21-46.

[7] - On the Hamkins approximation property, Ann. Pure Appl. Logic 144 (2006), 126-129.

[8] S. Shelah, Proper and Improper Forcing, Springer, 1998.

Department of Mathematics

University of California

Berkeley, CA 94720, U.S.A.

E-mail: jkrueger@math.berkeley.edu

http://www.math.berkeley.edu/ jkrueger

Received 3 December 2006;

in revised form 1 July 2008 